\title{
Piomiositis bilateral recurrente secundaria a embolismo séptico por endocarditis con cultivos negativos
}

\author{
Reporte de un caso
}

Gallego-Hermosillo Luis Armando*, Rivera-Castorena Ana Yoatzin*, Mojarro-Cisneros Miguel Ángel*, Torres-Cisneros Esaú Nefthaly*, González-Gámez Mario**, Dueñas-Silva Héctor Eduardo***

\section{Resumen}

- La piomiositis es una infección purulenta del músculo esqueléti-

- co que surge por diseminación hematógena, generalmente con

- formación de abscesos. Es clásica de los trópicos, aunque se ha

- reconocido en climas templados con frecuencia creciente.Típica-

- mente, comienza con dolor y leve edema de los músculos involu-

- crados, los cuales se vuelven cada vez más sensibles e indurados.

- El Staphylococcus aureus es la causa más común de piomiositis;

- causa hasta el 90 por ciento de los casos tropicales y hasta el

- 75 por ciento del resto de los casos. La endocarditis infecciosa

- (EI) se refiere a la infección de la superficie endocárdica del co-

- razón. Las tres causas más comunes de El en todo el mundo son

- los estafilococos, estreptococos y enterococos; sin embargo, en

- algunos casos la identificación del microorganismo responsable

- representa un reto para el médico tratante. La El con hemocul-

- tivos negativos se define como endocarditis sin etiología tras la

- inoculación de al menos tres muestras de sangre independientes

- en un sistema de hemocultivo estándar con cultivos negativos

- después de siete días de incubación y subcultivo. En el presen-

- te reporte se comunica el caso clínico de un paciente de 47

- años con piomiositis bilateral recurrente secundaria a embolismo

- séptico por endocarditis con cultivos negativos y se realiza una

- extensa revisión de la literatura. Se concluye que la piomiositis

- representa una condición pobremente comprendida que requiere

- un importante nivel de sospecha sobre todo en pacientes con

- alguna forma de inmunocompromiso. LUXMÉDICA AÑO 16, NÚMERO

- 47, MAYO-AGOSTO 2021, PP 61-72.

Palabras clave: piomiositis, embolización séptica, endocarditis infecciosa, endocarditis infecciosa con hemocultivos negativos.

\begin{abstract}
Pyomyositis is a purulent infection of the skeletal muscle that arises from hematogenous spread, usually with abscess formation. It is a classic of the tropics, although it has been recognized in temperate climates with increasing frequency. Typically, it begins with pain and mild swelling of the involved muscles, which become increasingly tender and indurated. Staphylococcus aureus is the most common cause of pyomyositis; it causes up to $90 \%$ of tropical cases and up to $75 \%$ of all other cases. Infective endocarditis (IE) refers to infection of the endocardial surface of the heart. The three most common causes of IE worldwide are staphylococci, streptococci, and enterococci. However, in some cases, identifying the responsible microorganism represents a challenge for the treating physician. Blood culture-negative IE is defined as endocarditis without etiology after inoculating at least three independent blood samples into a standard culture-negative blood culture system after seven days of incubation and subculture. In this report, the clinical case of a 47-year-old patient with recurrent bilateral pyomyositis secondary to septic embolism due to culture-negative endocarditis is reported, and an extensive review of the literature is carried out. Pyomyositis represents a poorly understood condition that requires a significant level of suspicion, especially in patients with some form of immunocompromise. LUXMÉDICA AÑO 16, NÚMERO 47, MAYO-AGOSTO 2021, PP 61-72.
\end{abstract}

* Médico Pasante de Servicio Social de la Universidad Autónoma de Aguascalientes. https://orcid.org/0000-0002-7592-4676. Correo electrónico armando_ gallego@hotmail.com

Médico Pasante de Servicio Social de la Universidad Autónoma de Aguascalientes. https://orcid.org/0000-0003-1312-3512. Correo electrónico anayo_ riverc@hotmail.com

* Médico Pasante de Servicio Social de la Universidad Autónoma de Aguascalientes. https://orcid.org/0000-0002-2047-9421. Correo electrónico mojarrocisneros@gmail.com

Médico Pasante de Servicio Social de la Universidad Autónoma de Aguascalientes. https://orcid.org/0000-0002-2477-5922. Correo electrónico esautorresc@gmail.com

**_Médico Internista-Infectólogo, adscrito al Centenario Hospital Miguel Hidalgo https://orcid.org/0000-0002-5776-3776. Correo electrónico dr.mariogzg@ gmail.com

*** Médico Internista, Adscrito al Hospital General de Zona No. 3 IMSS https://orcid.org/0000-0003-3157-9505 Correo electrónico drduenassilva@hotmail.com Fecha de recibido: 26 de septiembre 2020 Fecha de aceptación: 2 de marzo 2021 


\section{Introducción}

La endocarditis infecciosa (EI) puede adquirirse en la comunidad o en el contexto de exposición a la atención médica. ${ }^{1}$ Entre 2000 y 2011, la incidencia de El en los Estados Unidos aumentó de 11 por 100,000 habitantes a 15 por 100,000 habitantes. 2,3 Varios factores predisponen al desarrollo de $\mathrm{El}$, entre los que destacan la edad mayor a 60 años, sexo masculino, uso de drogas inyectables, dentición pobre o infección dental. ${ }^{4-14}$ Las manifestaciones clínicas de la El son variables; puede presentarse como una infección aguda, rápidamente progresiva o como una enfermedad subaguda o crónica con fiebre leve y síntomas inespecíficos. El diagnóstico se establece con base en manifestaciones clínicas, hemocultivos u otros datos microbiológicos y ecocardiografía. ${ }^{15}$ Los criterios aceptados para el diagnóstico de El son los criterios de Duke modificados (tabla 1).

La El es un desafío diagnóstico en algunos pacientes. La proporción de El sin diagnóstico etiológico varía de un país a otro y entre los diferentes centros en el mismo país. La El con hemocultivos negativos se define como endocarditis sin etiología tras la inoculación de al menos tres muestras de sangre independientes en un sistema de hemocultivo estándar con cultivos negativos después de siete días de incubación y subcultivo. ${ }^{16}$ Se pensaba tradicionalmente que los organismos HACEK (Aggregatibacter aphrophilus [anteriormente llamado Haemophilus aphrophilus] y Aggregatibacter paraphrophilus; Aggregatibacter actinomycetemcomitans; Cardiobacterium hominis; Eikenella corrodens; y Kingella kingae) eran los agentes más comunes de endocarditis con cultivo negativo. Sin embargo, los estudios han encontrado que los organismos HACEK pueden aislarse fácilmente con los sistemas actuales de hemocultivo cuando se incuban durante al menos cinco días. ${ }^{17,18}$

\section{Tabla I}

\section{Criterios de Duke modificados para el diagnóstico de (EI) ${ }^{19}$}

\section{Criterios de Duke modificados para el diagnóstico de endocarditis infecciosa (EI)19}

\section{Criterios mayores}

Hemocultivos positivos para El: 2 hemocultivos separados con aislamiento de microorganismos típicos para El (S. aureus, S. viridans, S. bovis, grupo HACEK).

Un cultivo positivo para C. burnetii o estudio serológico con anticuerpos positivos con títulos mayores a 1:800.

Evidencia de afectación endocárdica con ecocardiograma (vegetación, absceso o nueva dehiscencia de prótesis valvular), nuevo soplo cardíaco o cambio en uno preexistente.

\section{Criterios menores}

Predisposición: uso de drogas intravenosas o presencia de afección cardíaca predisponente.

Fiebre mayor o igual a $38^{\circ} \mathrm{C}\left(100.4^{\circ} \mathrm{F}\right)$.

Fenómenos vasculares: embolia arterial mayor, infartos sépticos pulmonares, aneurisma micótico, hemorragia intracraneal, hemorragias conjuntivales o lesiones de Janeway.

Fenómenos inmunológicos: Glomerulonefritis, ganglios de Osler, manchas de Roth o factor reumatoide.

Evidencia microbiológica: Hemocultivos positivos que no cumplen con los criterios principales o evidencia serológica de infección activa con un organismo compatible con El. 
En general, la terapia para la endocarditis infecciosa (EI) debe estar dirigida al organismo aislado en los hemocultivos; los cultivos son positivos en más del $90 \%$ de los pacientes con El. La elección de la terapia empírica debe tener en cuenta los patógenos más probables. En general, la terapia empírica debe cubrir Staphylococcus aureus (meticilina susceptible y resistente), Streptococcus sp. y Enterococcus $\mathrm{sp}$. La terapia se selecciona de acuerdo con el microorganismo involucrado, características del huésped y tasa de resistencias en la comunidad, usualmente consistente de un antimicrobiano betalactámico con vancomicina. Una vez conocida la susceptibilidad, se ajusta esquema de tratamiento, agregando medicamentos que aumenten la capacidad bactericida o aumenten su acción en biopelículas (aminoglucósidos, rifampicina). En general, la duración sugerida de la terapia en pacientes con endocarditis valvular nativa (NVE) varía hasta seis semanas. 7,20-23

La piomiositis es una infección purulenta del músculo esquelético que surge de la diseminación hematógena de una infección distante, generalmente con formación de abscesos. La piomiositis es una infección clásica de los trópicos, aunque se ha reconocido en climas templados con frecuencia creciente. ${ }^{20,21}$ Los factores predisponentes para piomiositis incluyen inmunodeficiencia (infección por el virus de la inmunodeficiencia humana, diabetes mellitus, cirrosis, malignidad, insuficiencia renal), trauma, uso de drogas intravenosas, infección concurrente, y la malnutrición.

El Staphylococcus aureus es la causa más común de piomiositis; causa hasta el $90 \%$ de los casos tropicales y hasta el 75 $\%$ del resto de casos. ${ }^{2,22-27}$ El diagnóstico temprano de piomiositis es difícil porque el músculo inflamado suele ser profundo, y los signos inflamatorios clásicos a menudo están ausentes en los tejidos blandos cir- cundantes. ${ }^{28}$ Las herramientas para el diagnóstico de la piomiositis incluyen estudios de imagen, cultivos y datos de laboratorio. Si está disponible, la resonancia magnética (MRI) es la técnica de imagen óptima. ${ }^{29,} 30$ Es muy sensible a la inflamación muscular, incluso antes de la formación de un absceso franco, y puede demostrar el grado de implicación en los tejidos.

La piomiositis se puede dividir en tres etapas clínicas ${ }^{27}$ : La etapa 1 se caracteriza por dolor muscular local con espasmos musculares, edema y fiebre de bajo grado, induración del músculo afectado y leucocitosis leve también pueden estar presentes. La etapa 2 ocurre de 10 a 21 días después del inicio de los síntomas y se caracteriza por fiebre, sensibilidad muscular exquisita y edema. Un absceso franco puede ser clínicamente evidente, y con la aspiración percutánea del músculo afectado generalmente se encuentra material purulento. ${ }^{31}$ La etapa 3 se caracteriza por toxicidad sistémica. El músculo afectado es fluctuante. Pueden ocurrir complicaciones de la bacteriemia por $S$. aureus como choque séptico, endocarditis, embolia séptica, neumonía, pericarditis, artritis séptica, absceso cerebral e insuficiencia renal aguda. ${ }^{32,33}$ La rabdomiólisis también se ha descrito. ${ }^{34}$ Aunque la piomiositis en etapa 1 se puede tratar con antibióticos solos, la mayoría de los pacientes presentan enfermedad en etapa 2 o 3 y, por lo tanto, requieren antibióticos y drenaje para el tratamiento definitivo. ${ }^{35}$ La duración de la terapia antimicrobiana debe adaptarse a la mejoría clínica y radiográfica. Por lo general, tres a cuatro semanas de terapia parenteral son suficientes.

El objetivo de este artículo es presentar un caso de piomiositis bilateral recurrente secundaria a embolismo séptico por endocarditis con cultivos negativos, el abordaje diagnóstico, el tratamiento y la revisión bibliográfica. 


\section{Presentación de caso clínico}

Se presenta el caso de un paciente de 47 años. Los antecedentes de interés son: carga genética para Diabetes mellitus tipo 2 e Hipertensión arterial sistémica; antecedentes personales patológicos: alergia a penicilina y sulfonamidas. Niega enfermedades crónico-degenerativas, antecedentes quirúrgicos, transfusionales o traumáticos. Comienza su padecimiento en septiembre de 2019 con cuadro clínico caracterizado por dolor punzante en región de muslo derecho en toda su extensión, el cual aumenta de manera progresiva hasta que dificulta la marcha; por lo que decide acudir a Hospital General de Zona, en donde se decide internamiento. Se realizó USG Doppler (Imagen 1) de miembro inferior derecho por sospecha de trombosis venosa profunda, la cual se descarta.

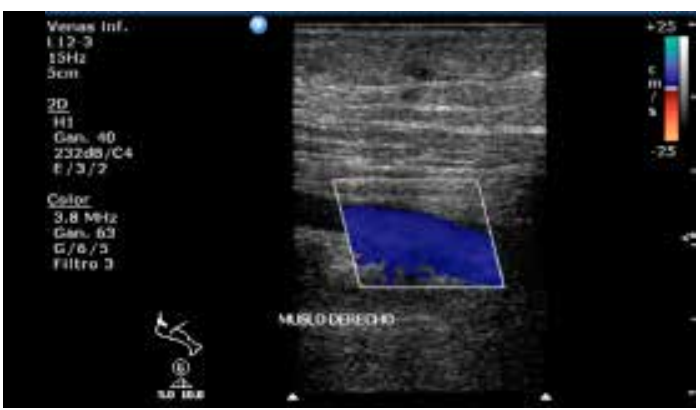

Imagen 1. USG doppler de muslo derecho: Mostró estudio negativo para trombosis venosa en muslo.

Se realizó también ultrasonografía de tejidos blandos de muslo derecho (Imáge- nes 2 y 3), en el cual se encontraron datos de miositis probablemente infecciosa de predominio a nivel de la unión miotendinosa del vasto intermedio, edema de tejidos blandos. Se realizó resonancia magnética contrastada (Imágenes 4 y 5), donde se evidenció cambios inflamatorios del cuádriceps derecho compatibles con miositis con aparente presencia de colección de 7.7 $\mathrm{mL}$ de tercio medio del vasto lateral (de la cual no se realizó cultivo), edema interfascial que circunda el trayecto del vasto anterior y vasto lateral, además de edema de tejidos blandos.
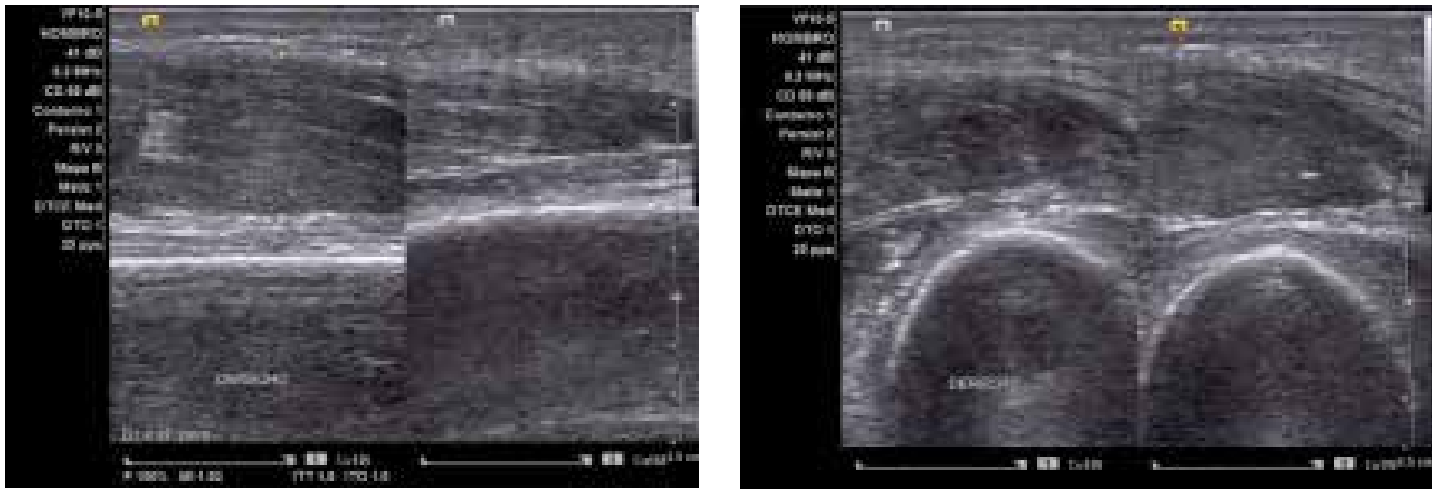

Imágenes 2 y 3. Ultrasonido de tejidos blandos de muslo derecho: muestra edema de tejidos blandos y datos compatibles con miositis probablemente infecciosa. 

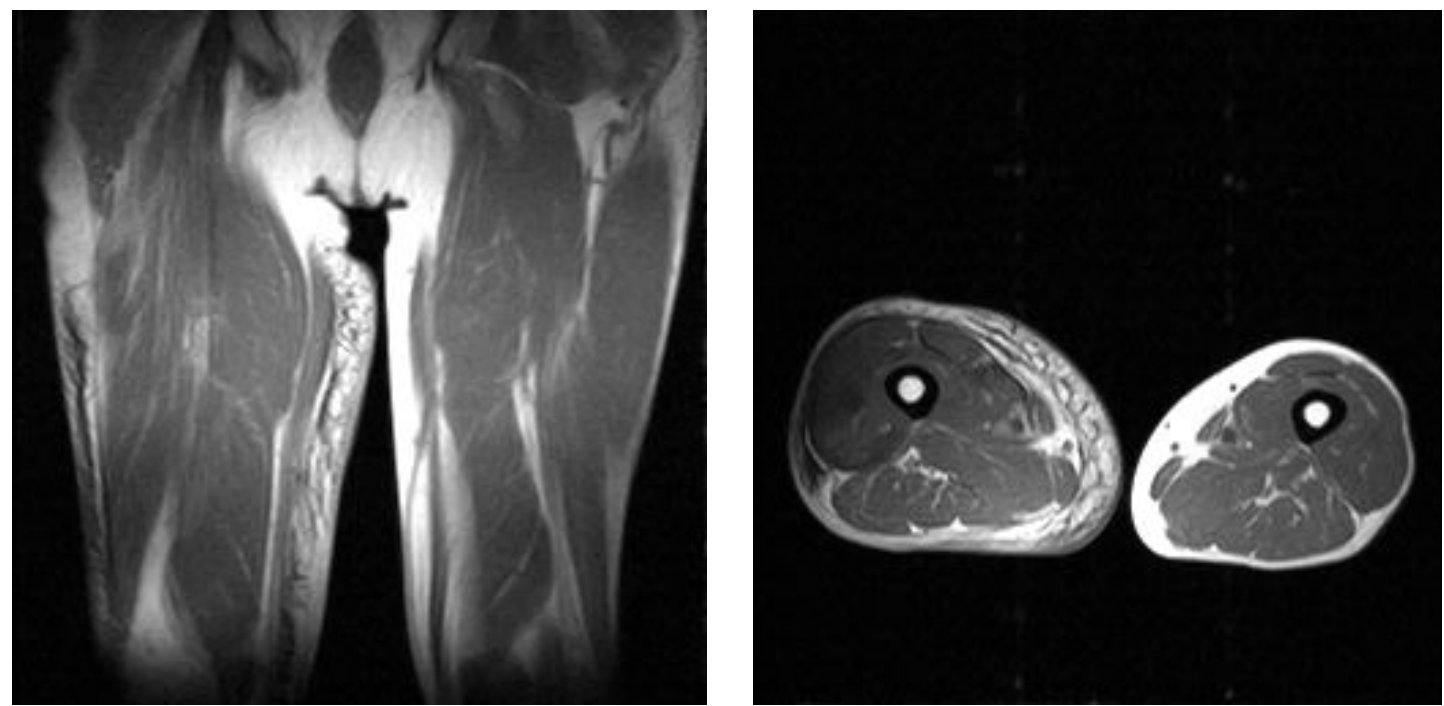

Imágenes 4 (corte coronal) y 5 (corte axial). Resonancia magnética contrastada: se observan cambios inflamatorios del cuádriceps derecho, compatibles con miositis con aparente presencia de colección de 7.7 cc de tercio medio del vasto lateral.

Se decidió realizar biopsia de muslo derecho tipo Tru-cut, guiada por ultrasonido (Imagen 6), con reporte histopatológico de músculo esquelético con zonas que muestran fibroblastos y vasos sanguíneos dilatados, además de infiltrado inflamatorio a expensas de neutrófilos y escasos linfocitos, con zonas donde se observan microabscesos, no se identifican elementos neoplásicos malignos.

Por lo anterior, se integró diagnóstico de piomiositis y se prescribe tratamiento antibiótico intrahospitalario con ceftriaxona vía intravenosa durante 10 días. Presentó discreta mejoría clínica y de marcadores inflamatorios, por lo cual se decidió su egreso hospitalario y se continuó con esquema antimicrobiano oral con ciprofloxacino 500 mg cada 12 horas por 5 días y linezolid 600 mg cada 12 horas por 10 días; con aparente remisión completa del cuadro.

Por otro lado, durante la hospitalización se estableció el diagnóstico de diabetes mellitus tipo 2, por cifras de glucemia persistentemente altas y hemoglobina glucosilada compatible. Se realizó diagnóstico además de hipertensión arterial sistémica durante hospitalización.

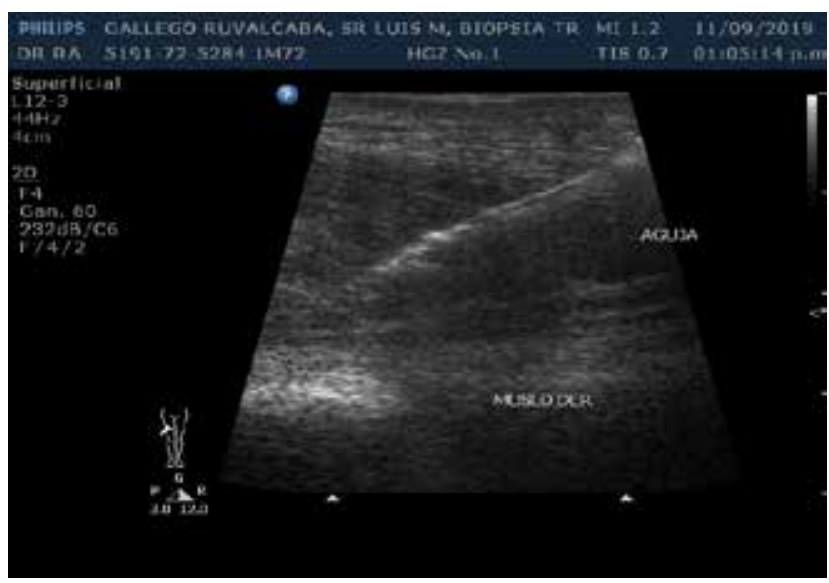

Imagen 6. Biopsia de muslo derecho tipo Trucut guiada por ultrasonido: Se observa la aguja entre el tejido muscular inflamado. 
Cinco meses después, (febrero del 2020), el paciente presentó otro cuadro clínico similar al previo, caracterizado por una semana de evolución con dolor punzante en muslo de extremidad inferior izquierda, así como en región medial de muslo de pierna derecha, de intensidad grave, progresivo, que dificultaba la marcha, así como la flexo-extensión de las extremidades, que aumentaba con la palpación, y no presentaba fiebre; sin embargo, se observó edema de predominio en muslo izquierdo. Acudió a valoración médica en donde, además de lo ya comentado, se advierte hipotensión arterial, por lo que se decidió hospitalización.

A su ingreso, se le realizaron estudios de laboratorio que evidenciaron aumento en enzimas musculares CPK $563 \mathrm{U} / \mathrm{I}$, mioglobina $218.2 \mathrm{ng} / \mathrm{ml}$ y de reactantes de fase aguda con PCR $99.4 \mathrm{mg} / \mathrm{l}$ VSG $37 \mathrm{~mm} /$ hr. Se agregó a sintomatología, fiebre de hasta $38.2{ }^{\circ} \mathrm{C}$, asociado a escalofríos. De igual manera, a su ingreso se realizaron dos hemocultivos periféricos $y$, posteriormente, se inició un tratamiento empírico con ceftriaxona y vancomicina.

Se realizó un ecocardiograma transtorácico (ECOTT) (Imagen 7) que evidenció masa filiforme delgada hiperecogénica en válvula mitral adherida al margen de la valva anterior con movimiento caótico, errante de aproximadamente $6 \mathrm{~mm} \times 1 \mathrm{~mm}$ consistente con vegetación, sin datos de falla cardíaca.

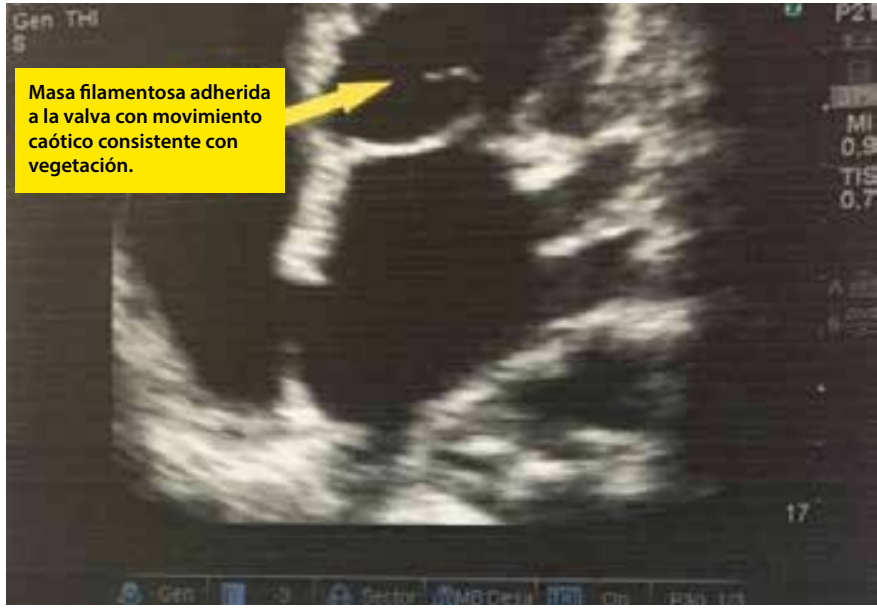

Imagen 7. ECOTT: Se observa vegetación en válvula mitral adherida al margen de la valva anterior. Sin datos de falla cardíaca.

Se realizó nueva resonancia magnética de abdomen y ambos muslos (Imágenes 8 y 9), evidenciando discreta hepatomegalia, nódulos linfáticos retroperitoneales y paraaórticos de aspecto reactivo, incremento de volumen y edema de los músculos aductor mayor, menor y medio derechos en su tercio proximal y medio, porción media y distal de la cabeza larga del bíceps crural derecho, porción media del sartorio derecho, vasto medial izquierdo, aductor magno izquierdo, porción media del sartorio izquierdo y en menor proporción de la porción media del gracilis ipsilateral, con restricción en la secuencia de difusión y reforzamiento heterogéneo en el contraste intravenoso que sugirió miositis infecciosa, sin lograr identificar colecciones organizadas. 


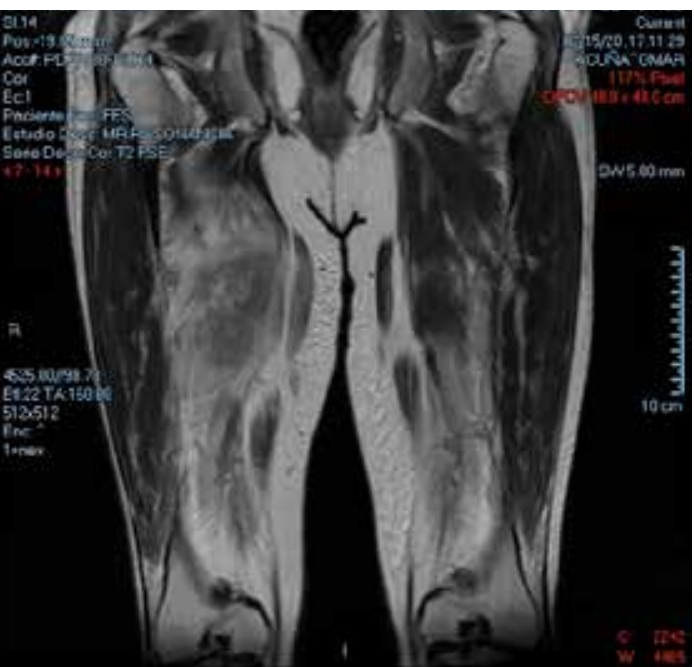

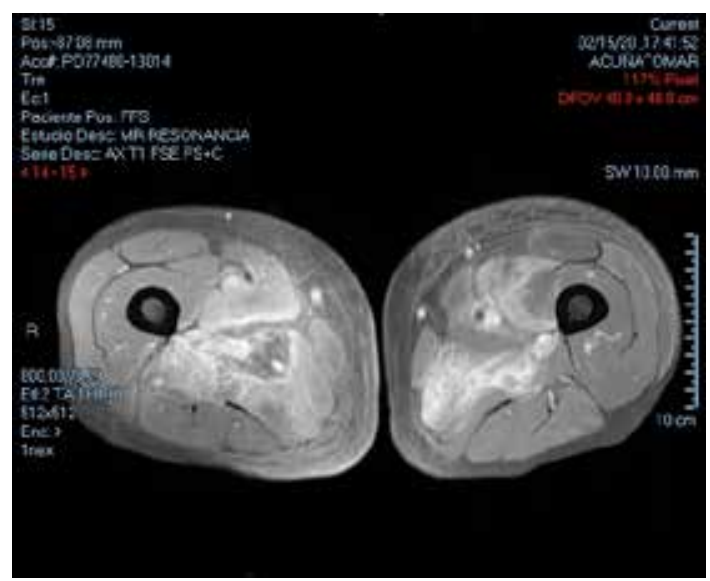

Imágenes 8 (corte coronal) y 9 (corte axial).

Resonancia magnética contrastada: se observa

edema de músculos del cuádriceps de forma bilateral sin presencia de colecciones organizadas.

Se realizó además biopsia quirúrgica (Imágenes 10, 11 y 12) del músculo cuádriceps izquierdo, que reportó músculo estriado con áreas irregulares y multifocales de necrosis isquémica que borra las estriaciones y en algunas zonas se extiende al tejido adiposo vecino, en algunas áreas se observa infiltrado inflamatorio linfocitario en disposición perivascular sin infiltración de la pared vascular. Hallazgos que pudieran ser compatibles con embolismo sépti$c o$. Se realizaron tinciones de Gram, ZiehlNeelsen y PAS, las cuales no demostraron la presencia de organismos específicos. En el cultivo de biopsia de músculo no hubo desarrollo bacteriano.

Por otro lado, durante su estancia hospitalaria se realizaron dos hemocultivos periféricos más. Los cuatro hemocultivos (dos antes y dos después de iniciar tratamiento empírico) se incubaron durante 14 días, siendo reportados al final sin desarrollo. Se concluyó que no se contó con aislamiento microbiológico en últimos dos hemocultivos por inicio de tratamiento antimicrobiano previo a su toma.

Como parte del abordaje diagnóstico de endocarditis infecciosa con cultivos negativos, se decidió realizar al paciente la prue- ba rosa de bengala junto con la prueba de aglutinación en suero (Huddleson) para la detección de Brucella sp., en ambas pruebas el resultado fue negativo, descartando dicha posibilidad. Se realizaron anticuerpos anti-nucleares y anti-ADN de doble cadena, con la intención de descartar enfermedad autoinmune, con resultados negativos.

Como resultado del estudio diagnóstico previamente descrito, se integró como diagnóstico final: piomiositis bilateral recurrente secundaria a embolismo séptico por endocarditis infecciosa de válvula mitral nativa con cultivos negativos.

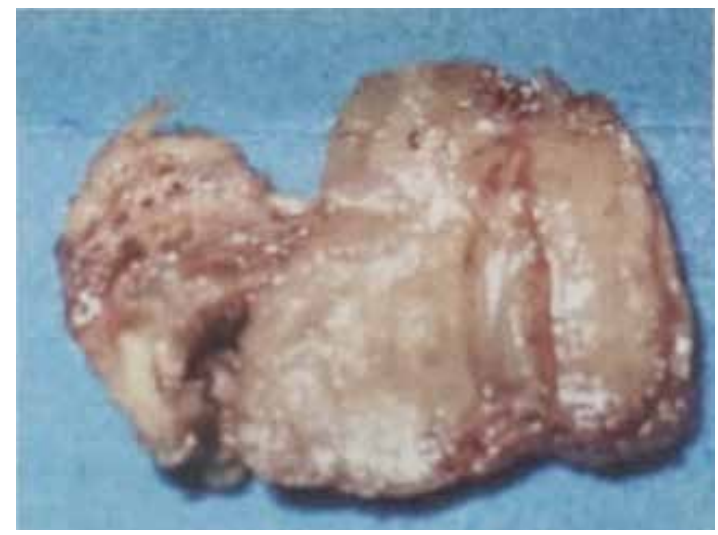

Imagen 10. Biopsia (pieza quirúrgica) de muslo izquierdo 

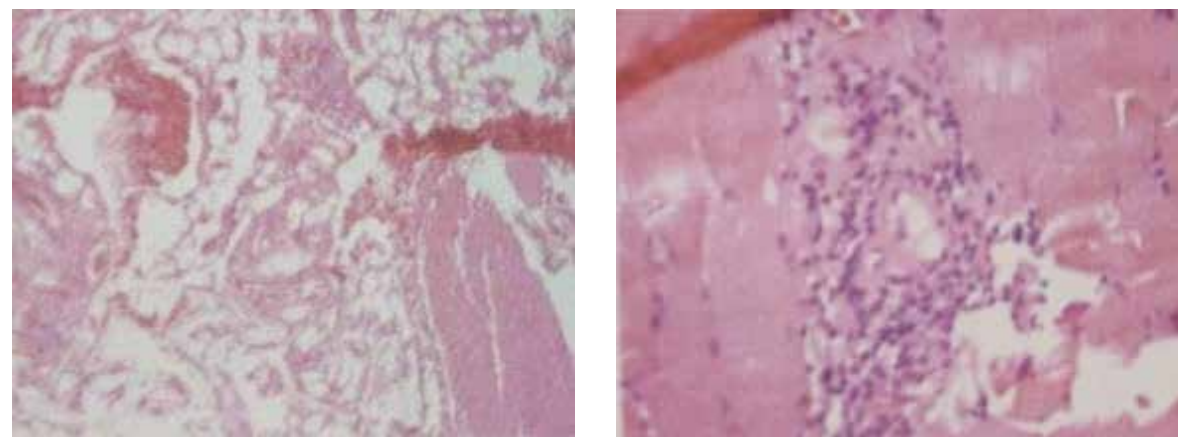

Imágenes 11 y 12. Estudio histopatológico de cuádriceps izquierdo: se observan áreas irregulares y multifocales de necrosis isquémica, así como infiltrado inflamatorio linfocitario en disposición perivascular sin infiltración de la pared vascular. Datos compatibles con embolismo séptico.

Como se comentó, desde su ingreso, y posterior a toma de dos hemocultivos, se le inició terapia empírica con doble esquema antimicrobiano vía intravenosa con ceftriaxona 1 gramo cada 12 horas y vancomicina 1.5 gramos $(15 \mathrm{mg} / \mathrm{kg} /$ dosis $)$ cada 12 horas. El tratamiento lo recibió durante 14 días. Se observó mejoría considerable de cuadro clínico con disminución de edema, dolor y resolución de fiebre.

Se realizó nuevo ecocardiograma el cual reportó la presencia de mismo filamento adherido a la valva anterior de la válvula mitral con movimiento ondulante y errático, con mayor dificultad para su visuali- zación ecocardiográfica, lo que sugirió una disminución en su tamaño.

También se observó mejoría en valores de laboratorio con disminución de enzimas musculares y reactantes de fase aguda. Se decidió alta hospitalaria con tratamiento antibiótico vía oral por cuatro semanas más con levofloxacino $750 \mathrm{mg}$ cada 24 horas y linezolid $600 \mathrm{mg}$ cada 12 horas.

En la revisión a 12 semanas posterior a suspensión de tratamiento, se encontró paciente con sintomatología completamente resuelta, sin dolor o edema en músculos afectados, sin fiebre y con reactantes de fase aguda negativos.

\section{| | | | | | | | | | | | | | | | | | | | | | | | | | | | | | | | | | | | | | | | | | | | | | | | | | | | | | | | | | | | | | | | | | | | | | | | | | | | | | | | | | | | | | | | | | | | | | | | | |}

\section{Discusión}

Nos pareció apropiado presentar este caso clínico por lo peculiar del cuadro clínico caracterizado por piomiositis bilateral, la cual es una presentación rara de la enfermedad, y por su asociación a endocarditis infecciosa como el sitio que originó la infección muscular.

La piomiositis bilateral secundaria a embolismo séptico por endocarditis infecciosa con cultivos negativos es una condición rara que requiere de un alto nivel de sospecha para realizar un abordaje diagnóstico adecuado desde el inicio de la enfermedad.
Usualmente en estos casos, los hombres parecen ser más frecuentemente afectados que las mujeres, además de que muchos cursan con estados de inmunocompromiso $u$ otras comorbilidades subyacentes. La diabetes mellitus, comorbilidad con la que debutó el paciente durante su ingreso inicial, se ha implicado como forma de inmunodeficiencia asociada con la piomiositis.

La enfermedad ocurre con mayor frecuencia en la extremidad inferior (los sitios incluyen los músculos del muslo, la pantorrilla y los glúteos), pero cualquier grupo muscular puede estar involucrado, inclui- 
dos los músculos iliopsoas, pélvicos, del tronco, paraespinales y de las extremidades superiores. ${ }^{2,36}$ La infección multifocal con afectación de más de un grupo muscular puede ser observada en hasta un $20 \%$ de los casos. ${ }^{2,37}$

En cuanto a la El, la fiebre es el síntoma más común (hasta el $90 \%$ de los pacientes la presenta); a menudo se asocia con escalofríos, anorexia y pérdida de peso. ${ }^{3}$ Síntomas presentados por el paciente en algún momento de su evolución.

Se ha observado que la El está asociada con una amplia gama de complicaciones sistémicas debido a la embolización séptica, que puede estar asociada con trombosis localizada, hemorragia, infección o desarrollo de reacciones inmunes. ${ }^{35}$ Las manifestaciones clínicas que reflejan estas complicaciones pueden estar presentes en el momento de la presentación inicial o pueden desarrollarse posteriormente.

La identificación del agente etiológico es crítica en la El para seleccionar un tratamiento apropiado, ya que la tasa de mortalidad sigue siendo alta. La epidemiología de la El con hemocultivos negativos varía según el país y el huésped; los organismos HACEK a menudo crecen en cinco días utilizando el sistema BACTEC; sin embargo, los cultivos siguen siendo negativos en el $2 \%$ al $7 \%$ de los pacientes con El, incluso cuando se toma el máximo cuidado para obtener la cantidad y el volumen adecuados de hemocultivos; ${ }^{38-40}$ en el caso de nuestro paciente, se tomaron cuatro hemocultivos de manera adecuada, dos de ellos en las primeras 12 horas de hospitalización, el tercero entre las 24 y 36 horas y el cuarto a las 48 horas de su ingreso hospitalario.

Los cultivos son negativos en la El por tres razones principales: 1) Administración previa de agentes antimicrobianos, 2) Técnicas microbiológicas inadecuadas y 3) Infección con bacterias altamente fastidiosas o patógenos no bacterianos (p. ej., hongos). En nuestro paciente, el doble es- quema antibiótico se inició después de la segunda toma de sangre para cultivo.

Los agentes causales más comunes de la endocarditis infecciosa con hemocultivo negativo (EI) son organismos fastidiosos ( $p$. ej., agentes zoonóticos y hongos) y Streptococcus sp. en pacientes que han recibido tratamiento antibiótico previo. ${ }^{41}$ Las diferencias en la frecuencia de El con cultivo negativo en los países reflejan la mayor incidencia de agentes zoonóticos fastidiosos (por ejemplo, Bartonella sp., Coxiella burnetii o Brucella sp.) que causan infección humana en los países en desarrollo; 42 en el caso de nuestro paciente, ante el resultado negativo de los cuatro hemocultivos, se realizó la búsqueda intencionada de Brucella, la cual resultó también negativa.

Las pruebas de diagnóstico para endocarditis con cultivo negativo incluyen técnicas especiales de cultivo (p. ej., hemocultivo con centrifugación de lisis), técnicas moleculares (p. ej., reacción en cadena de la polimerasa [PCR] y ensayos serológicos) y evaluación histopatológica del tejido valvular cuando se realiza la escisión quirúrgica. ${ }^{43,44}$ No se realizaron en el caso clínico presentado por no requerir tratamiento quirúrgico.

Para los pacientes con sospecha de EI que se presentan sin síntomas agudos, la terapia empírica no siempre es necesaria, y la terapia puede esperar resultados de hemocultivos. La terapia empírica debe cubrir Staphylococcus aureus (meticilina susceptible y resistente), especies de estreptococos y enterococos. Usualmente la combinación de un antimicrobiano betaláctamico con vancomicina (15 a $20 \mathrm{mg} / \mathrm{kg} /$ dosis cada 8 a 12 horas, sin exceder los $2 \mathrm{~g}$ por dosis) es una opción adecuada para la terapia empírica en la mayoría de los pacientes. ${ }^{38,39}$

El tratamiento empírico de pacientes con El con cultivos negativos debe abarcar tanto los organismos grampositivos como los gramnegativos; vancomicina y cefepima como régimen inicial para pacientes con presentación clínica aguda es una buena 
opción, para los pacientes con una presentación clínica subaguda la cobertura empírica podría incluir vancomicina y ampicilinasulbactam como régimen inicial. ${ }^{38,39}$

La terapia posterior debe dirigirse al microorganismo específico si las pruebas de diagnóstico, como la reacción en cadena de la polimerasa o la serología, identifican el agente etiológico. La mayoría de los pacientes con El se vuelven afebriles de tres a cinco días después del inicio de la terapia antimicrobiana adecuada.

En cuanto al tratamiento de la piomiositis, para las personas inmunocompetentes, la terapia antibiótica parenteral empírica inicial debe dirigirse contra especies de estafilococos, incluidos S. aureus resistente a la meticilina (MRSA) y estreptococos. ${ }^{1}$ Para las personas inmunocomprometidas, se debe administrar una amplia cobertura de antibióticos para los organismos gram- positivos, gramnegativos y anaerobios. En tales circunstancias, la vancomicina puede combinarse con un régimen de amplio espectro que tenga actividad contra gramnegativos y anaerobios; nuestro paciente tiene el diagnóstico de diabetes mellitus tipo 2 desde septiembre de 2019; al tratarse de un paciente inmunocomprometido, y con la intención de cubrir los estafilococos y los estreptococos como microorganismos principales causantes de piomiositis, recibió doble esquema antibiótico vía intravenosa durante su hospitalización con ceftriaxona y vancomicina, con notable mejoría clínica y de laboratorio. Tras su egreso hospitalario se continuó con doble esquema antibiótico por vía oral a base de levofloxacino y linezolid, con la intención de mantener espectro contra bacterias gram negativas y gram positivas (principalmente Staphylococcus aureus).

\section{Conclusiones}

1.- La piomiositis es una condición poco entendida que puede ser fatal si no se diagnostica de forma temprana y que requiere un alto nivel de sospecha, sobre todo en pacientes con diabetes mellitus y/o con otro tipo de inmunocompromiso.

2.- La piomiositis secundaria a embolismo séptico por endocarditis infecciosa es una condición rara, anteriormente descrita en pacientes con uso de drogas por vía intravenosa.

3.- Es importante un abordaje diagnóstico completo de la piomiositis para determinar la causa exacta de la misma, siempre teniendo un alto índice de sospecha.

4.- Es importante conocer, y tener en cuenta como posible causa de piomiositis, el diagnóstico de endocarditis infecciosa (EI), aún cuando los cultivos realizados sean negativos. El tener dicho conocimiento es esencial para un diagnóstico temprano y un adecuado manejo, disminuyendo así las posibles complicaciones y logrando un mejor pronóstico para el paciente.

\section{Bibliografía}

1.- N. Deborah Friedman, Keith S. Kaye, Jason E. Stout, Sarah A. McGarry, Sharon L. Trivette, Jane P. Briggs, Wanda Lamm, Connie Clark, Jennifer MacFarquhar, Aaron L. Walton, L. Barth Reller, Daniel J. Sexton. Health care--associated bloodstream infections in adults: a reason to change the accepted definition of community-acquired infections. Annals of Internal Medicine,2002;137 (10):791-797.
2.- Sadip Pant, Nileshkumar J. Patel, Abhishek Deshmukh, Harsh Golwala, Nilay Patel, Apurva Badheka, Glenn A. Hirsch, Jawahar L. Mehta. Trends in infective endocarditis incidence, microbiology, and valve replacement in the United States from 2000 to 2011. Journal of the American College of Cardiology,2015;65(19): 2070-2076.

3.- Nana Toyoda, Joanna Chikwe, Shinobu Itagaki, Annetine C. Gelijns, David H. Adams, Natalia N. Egorova. Trends 
in Infective Endocarditis in California and New York State, 1998-2013. JAMA, 2017;317(16):1652-1660.

4.- Evelyn E. Hill, Paul Herijgers, Piet Claus, Steven Vanderschueren, Marie-Christine Herregods, Willy E. Peetermans. Infective endocarditis: changing epidemiology and predictors of 6-month mortality: a prospective cohort study. European Heart Journal, 2007; 28(2):196-203.

5.- Cantrell, M., \& Yoshikawa, T. T. Infective endocarditis in the aging patient. Gerontology, 1984; 30(5): 316-326.

6.- Francisco J. Castillo, Manuel Anguita, Juan C. Castillo, Martín Ruiz, Dolores Mesa, José Suárez de Lezo. Changes in Clinical Profile, Epidemiology and Prognosis of Leftsided Native-valve Infective Endocarditis Without Predisposing Heart Conditions. Revista Española de Cardiología (English Edition), 2015; 68(5): 445-448.

7.- Phillip I. Lerner, Louis Weinstein.Infective endocarditis in the antibiotic era. New England Journal of Medicine, 1966; 274(4): 199-206.

8.- C. Watanakunakorn. Changing epidemiology and newer aspects of infective endocarditis. Advances in Internal Medicine, 1977; 22: 21-47.

9.- Peter B. Lockhart, Michael T. Brennan, Martin Thornhill, Bryan S. Michalowicz, Jenene Noll, Farah K. Bahrani-Mougeot, Howell C. Sasser. Poor oral hygiene as a risk factor for infective endocarditis-related bacteremia. The Journal of the American Dental Association, 2009; 140(10), 12381244.

10.-Su-Jung Chen, Chia-Jen Liu, Tze-Fan Chao, Kang-Ling Wang, Fu-Der Wang, Tzeng-Ji Chen, Chern-En Chiang. Dental scaling and risk reduction in infective endocarditis: a nationwide population-based case-control study Canadian Journal of Cardiology, 2013; 29(4): 429-433.

11.-Walter Wilson, Kathryn A. Taubert, Michael Gewitz, Peter B. Lockhart, Larry M. Baddour, Matthew Levison, Ann Bolger, Christopher H. Cabell, Masato Takahashi, Robert S. Baltimore, Jane W. Newburger, Brian L. Strom, Lloyd Y. Tani, Michael Gerber, Robert O. Bonow, Thomas Pallasch, Stanford T. Shulman, Anne H. Rowley, Jane C. Burns, Patricia Ferrieri, Timothy Gardner, David Goff, David T. Durack. Prevention of infective endocarditis: guidelines from the American Heart Association: a guideline from the American Heart Association Rheumatic Fever, Endocarditis, and Kawasaki Disease Committee, Council on Cardiovascular Disease in the Young, and the Council on Clinical Cardiology, Council on Cardiovascular Surgery and Anesthesia, and the Quality of Care and Outcomes Research Interdisciplinary Working Group. Circulation, 2007; 116(15): 1736-1754

12.-F. K. Gould, T. S. J. Elliott, J. Foweraker, M. Fulford, J. D. Perry, G. J. Roberts, J. A. T. Sandoe, R. W. Watkin. Guidelines for the prevention of endocarditis: report of the Working Party of the British Society for Antimicrobial Chemotherapy. Journal of Antimicrobial Chemotherapy, 2006; 57(6): 1035-1042.

13.- Brian L. Strom, Elias Abrutyn, Jesse A. Berlin, Judith L. Kinman, Roy S. Feldman, Paul D. Stolley, Matthew E. Levison, Oksana M. Korzeniowski, Donald Kaye. Risk factors for infective endocarditis: oral hygiene and nondental exposures. Circulation, 2000; 102(23): 2842-2848.

14.-Xavier Duval, Sarah Millot, Catherine Chirouze, Christine Selton-Suty, Vanessa Moby, Pierre Tattevin, Christophe Strady, Edouard Euvrard, Nelly Agrinier, Daniel
Thomas, Bruno Hoen, François Alla, for the Eldents Association pour l'Etude et la Prévention de I'Endocardite Infectieuse (AEPEI) Study Group. Oral Streptococcal Endocarditis, Oral Hygiene Habits, and Recent Dental Procedures: A Case-Control Study. Clinical Infectious Diseases, 2017; 64(12): 1678-1685.

15.-Thomas J. Cahill, Bernard D. Prendergast. Infective endocarditis. The Lancet, 2016; 387(10021): 882-893.

16. D. Raoult, J., P. Casalta, H. Richet, M. Khan, E. Bernit, C. Rovery, S. Branger, F. Gouriet, G. Imbert, E. BotheIlo, F. Collart, G. Habib. Contribution of systematic serological testing in diagnosis of infective endocarditis. Journal of Clinical Microbiology, 2005; 43(10): 5238-5242.

17.- Ellen Jo Baron, John D. Scott, Lucy S. Tompkins. Prolonged incubation and extensive subculturing do not increase recovery of clinically significant microorganisms from standard automated blood cultures. Clinical Infectious Diseases, 2005; 41(11) : 1677-1680.

18.- Cathy A. Petti, Hasan S. Bhally, Melvin P. Weinstein, Kim Joho, Teresa Wakefield, L. Barth Reller, Karen C. Carroll. Utility of extended blood culture incubation for isolation of Haemophilus, Actinobacillus, Cardiobacterium, Eikenella, and Kingella organisms: a retrospective multicenter evaluation. Journal of Clinical Microbiology, 2006; 44(1): 257-259.

19.- Jennifer S. Li, Daniel J. Sexton, Nathan Mick, Richard Nettles, Vance G. Fowler, Jr., Thomas Ryan, Thomas Bashore, G. Ralph Corey. Proposed modifications to the Duke criteria for the diagnosis of infective endocarditis, Clinical Infectious Diseases, 2000; 30(4): 633-638.

20.- Nancy F. Crum. Bacterial pyomyositis in the United States. The American Journal of Medicine, 2004; 117(6): 420428.

21.-Paul Moriarty, Clare Leung, Mark Walsh, Clare Nourse. Increasing pyomyositis presentations among children in Queensland, Australia. The Pediatric Infectious Disease Journal, 2015; 34(1): 1-4.

22.- Laurent Christin, George A. Sarosi. Pyomyositis in North America: case reports and review. Clinical Infectious Diseases, 1992; 15(4): 668-677.

23.-S. Chauhan, S. Jain, S. Varma, S. S. Chauhan. Tropical pyomyositis (myositis tropicans): current perspective. Postgraduate Medical Journal, 2004; 80(943): 267-270.

24.-Lorne N. Small, John J. Ross. Tropical and temperate pyomyositis. Infectious Disease Clinics of North America, 2005 ; 19(4): 981-989.

25.-William A. Schwartzman, Mark W. Lambertus, Charles A. Kennedy, Matthew Bidwell Goetz. Staphylococcal pyomyositis in patients infected by the human immunodeficiency virus. The American Journal of Medicine, 1991; 90(1): 595-600.

26.- Juan J. Gomez-Reino, Juan J. Aznar, Jose L. Pablos, Federico Diaz-Gonzalez, Armando Laffon. Nontropical pyomyositis in adults. Seminars in Arthritis and Rheumatism, 1994; 23 (6): 396-405.

27.- L. Chukwuma Chiedozi. Pyomyositis. Review of 205 cases in 112 patients. The American Journal of Surgery, 1979; 137(2): 255-259.

28.- Richard K. Gibson, Stanton J. Rosenthal, Barbara P. Lukert. Pyomyositis. Increasing recognition in temperate climates. The American Journal of Medicine, 1984; 77(4): 768-772. 29.-Dennis L. Stevens, Alan L. Bisno, Henry F. Chambers, E. 
Patchen Dellinger, Ellie J. C. Goldstein, Sherwood L. Gorbach, Jan V. Hirschmann, Sheldon L. Kaplan, Jose G. Montoya, James $C$. Wade. Practice guidelines for the diagnosis and management of skin and soft tissue infections: 2014 update by the infectious diseases society of America. Clinical Infectious Diseases, 2014; 59(2): e10-e52.

30.-Stavroula J. Theodorou, Daphne J. Theodorou, Donald Resnick. MR imaging findings of pyogenic bacterial myositis (pyomyositis) in patients with local muscle trauma: illustrative cases. Emergency Radiology, 2007; 14(2): 89-96.

31.-W. R. C. Peckett, A. Butler-Manuel, L. A. Apthorp. Pyomyositis of the iliacus muscle in a child. The Journal of Bone and Joint Surgery, 2001; 83(1): 103-105.

32.- Clare Nourse, Mike Starr, Wendy Munckhof. Communityacquired methicillin-resistant Staphylococcus aureus causes severe disseminated infection and deep venous thrombosis in children: literature review and recommendations for management. Journal of Paediatrics and Child Health, 2007; 43(10):656-661.

33.- Michael Y. Lin, Katayoun Rezai, David N. Schwartz. Septic pulmonary emboli and bacteremia associated with deep tissue infections caused by community-acquired methiciIlin-resistant Staphylococcus aureus. Journal of Clinical Microbiology, 2008; 46(4):1553-1555.

34.- G. F. Falasca, A. J. Reginato. The spectrum of myositis and rhabdomyolysis associated with bacterial infection. The Journal of Rheumatology, 1994; 21(10):1932-1937.

35.-Bruno Hoen, Xavier Duval. Infective endocarditis. New England Journal of Medicine, 2013; 368(15):1425-1433.

36.- J. J. Shepherd. Tropical myositis: is it an entity and what is its cause?. The Lancet, 1983; 322(8361):1240-1242.

37.- Radouane Niamane, Ouassima Jalal, Meryem El Ghazi, Rachid Hssaida, Ali Had. Multifocal pyomyositis in an immunocompetent patient. Joint Bone Spine, 2004;71(6):595597.

38.- Larry M. Baddour, Walter R. Wilson, Arnold S. Bayer, Vance G. FowlerJr, Imad M. Tleyjeh, Michael J. Rybak, Bruno Barsic, Peter B. Lockhart, Michael H. Gewitz, Matthew E. Levison, Ann F. Bolger, James M. Steckelberg, Robert
S. Baltimore, Anne M. Fink, Patrick O'Gara, Kathryn A. Taubert. Infective Endocarditis in Adults: Diagnosis, Antimicrobial Therapy, and Management of Complications: A Scientific Statement for Healthcare Professionals From the American Heart Association. Circulation. 2015;132(15): 1435-1486.

39.-F. Kate Gould, David W. Denning, Tom S. J. Elliott, Juliet Foweraker, John D. Perry, Bernard D. Prendergast, Jonathan A. T. Sandoe, Michael J. Spry, Richard W. Watkin Guidelines for the diagnosis and antibiotic treatment of endocarditis in adults: a report of the Working Party of the British Society for Antimicrobial Chemotherapy. Journal of Antimicrobial Chemotherapy, 2012; 67(2): 269-289.

40.-Alan D. Tice, Susan J. Rehm, Joseph R. Dalovisio, John S. Bradley, Lawrence P. Martinelli, Donald R. Graham, R. Brooks Gainer, Mark J. Kunkel, Robert W. Yancey, David N. Williams. Practice Guidelines for Outpatient Parenteral Antimicrobial Therapy. Clinical Infectious Diseases, 2004; 38(12): 1651-1671.

41.- Pierre-Edouard Fournier, Franck Thuny, Hervé Richet, Hubert Lepidi, Jean-Paul Casalta, Jean-Pierre Arzouni, Max Maurin, Marie Célard, Jean-Luc Mainardi, Thierry Caus, Frédéric Collart, Gilbert Habib, Didier Raoult. Comprehensive Diagnostic Strategy for Blood Culture-Negative Endocarditis: A Prospective Study of 819 New Cases. Clinical Infectious Diseases, 2010; 51(2): 131-140.

42.-Akila Benslimani, Florence Fenollar, Hubert Lepidi, Didier Raoult. Bacterial zoonoses and infective endocarditis, Algeria. Emerging Infectious Diseases, 2005; 11(2): 216-224.

43.-D. Raoult, J. P. Casalta, H. Richet, M. Khan, E. Bernit, C. Rovery, S. Branger, F. Gouriet, G. Imbert, E. Bothello, F. Collart, G. Habib. Contribution of Systematic Serological Testing in Diagnosis of Infective Endocarditis. Journal of Clinical Microbiology, 2005; 43(10): 5238-5242.

44.- Pierre Houpikian, Didier Raoult. Diagnostic methods. Current best practices and guidelines for identification of difficult-to-culture pathogens in infective endocarditis. Cardiology Clinics, 2003; 21 (2): 207-217. 